\title{
Imaging Modalities for Hepatocellular Carcinoma Surveillance: Expanding Horizons beyond Ultrasound
}

\author{
Hyo Jung Park, So Yeon Kim \\ Department of Radiology and the Research Institute of Radiology, Asan Medical Center, University of Ulsan College of Medicine, Seoul, \\ Korea
}

Received Jan. 2, 2020

Revised Apr. 14, 2020

Accepted Apr. 16, 2020
In Asian countries favoring loco-regional treatment such as surgical resection or ablation, very early-stage hepatocellular carcinoma (HCC) should be the main target for surveillance. Even though ultrasound (US) has been accepted as a primary imaging modality for HCC surveillance, its performance in detecting very early-stage HCCs is insufficient. Moreover, in more than $20 \%$ of patients at high risk for HCC, visualization of the liver on US may be limited owing to the advanced distortion and heterogeneity of the liver parenchyma. Recently revised $\mathrm{HCC}$ clinical guidelines allow the use of alternative surveillance tools including computed tomography or magnetic resonance imaging in patients with inadequate US exams. This paper summarizes the findings of recent studies using imaging modalities other than US as surveillance tools for HCC as well as strengths and limitations of these modalities. (J Liver Cancer 2020;20:99-105)

Keywords: Hepatocellular carcinoma; Surveillance; Imaging

\section{INTRODUCTION}

Hepatocellular carcinoma (HCC) is the leading cause of death in patients with liver cirrhosis, largely due to failed early detection. ${ }^{1}$ Therefore, international guidelines recommend imaging-based HCC surveillance for patients at high risk. ${ }^{2-4}$ The treatment patterns for HCC vary across regions or countries according to the demographic characteristics, HCC prevalence, socioeconomic status, and medical resource availability. ${ }^{5}$ In Asian countries including Korea, surgical resection and image-guided ablation are preferred over de-

\section{Corresponding author : So Yeon Kim}

Department of Radiology and the Research Institute of Radiology, Asan Medical Center, University of Ulsan College of Medicine, 88 Olympic-ro 43-gil, Songpa-gu, Seoul 05505, Korea

Tel. +82-2-3010-4352, Fax. +82-2-476-0090

E-mail; sykim.radiology@gmail.com

https://orcid.org/0000-0001-6853-8577 ceased donor liver transplantation (DDLT), which is the main curative treatment option in Western countries. In this setting favoring loco-regional treatment, the main target for HCC surveillance differs from that in settings in which liver transplantation, especially DDLT, is the primary option. While DDLT as an HCC treatment requires high specificity comparable to that attained by histopathologic diagnosis to maximize organ utilization, ${ }^{6}$ loco-regional treatment emphasizes the importance of sensitive detection of HCC at very early stage. The outcomes of surgical resection ${ }^{7.8}$ and ablation $^{9-11}$ are closely related to HCC size, with $2 \mathrm{~cm}$ suggested as a size cut-off for HCC aggressiveness and invasiveness. ${ }^{7,12,13}$ Especially for radiofrequency ablation, the preferable size is smaller than $2 \mathrm{~cm}^{11}$ as the goal is to obtain a 360-degree, 0.5 $1.0 \mathrm{~cm}$ ablative margin all around the target tumor. ${ }^{14}$ Thus, in Asian countries favoring loco-regional treatment, very early-stage HCC should be the main target for surveillance. 
The goal of this article was to review the performance and limitations of ultrasound (US), to update readers regarding recent studies assessing imaging modalities other than US as possible HCC surveillance tools, and to summarize the strengths and limitations of each modality in detecting very early-stage HCC.

\section{ULTRASOUND: LIMITATIONS AS THE SOLE IMAGING MODALITY FOR HCC SURVEIL- LANCE}

Grayscale US is accepted as a primary imaging modality for HCC surveillance. Current clinical guidelines endorse surveillance using US with or without measurement of levels of serum tumor markers such as alpha-fetoprotein (AFP) every six months in populations at risk for HCC. ${ }^{2-4}$ However, it should be noted that US has limited sensitivity. ${ }^{15}$ A meta-analysis including 13 studies and 1,514 patients reported a US sensitivity of $63 \%$ and $69 \%$ for US with AFP in detecting early-stage HCC (one nodule $<5 \mathrm{~cm}$ or three nodules each $<3 \mathrm{~cm}$ in diameter) in patients with cirrhosis. ${ }^{16}$ Another recent meta-analysis with 15 studies and 4,400 patients reported sensitivity of US as low as $47 \%$ for early-stage HCC. ${ }^{17}$ For HCC smaller than $2 \mathrm{~cm}$, the sensitivity of US is further decreased; a recent prospective study reported a strikingly low US sensitivity for very early-stage HCC of 27.3\%. ${ }^{18}$ Given these disappointing results, a more sensitive surveillance tool is required to detect HCC at very early stages. Moreover, visualization of the liver and focal hepatic lesions on US differs between patients. Approximately 20-30\% of US examinations are classified as inadequate for HCC surveillance in patients at high risk of developing HCC, and inadequate US were significantly associated with advanced liver cirrhosis. ${ }^{1920}$ The sensitivity of US to detect hepatic lesions is also affected by lesion size and patient body status. A previous study ${ }^{21}$ showed that US sensitivity differed according to HCC size, as $85 \%$ for lesions $>4 \mathrm{~cm}, 65 \%$ for lesions $2-4 \mathrm{~cm}$, and $21 \%$ for lesions $<2 \mathrm{~cm}$. As obesity poses challenges for US to detect HCC due to limited sonic beam penetration, the sensitivity of US was only $21 \%$ in patients with body mass index (BMI) $\geq 30 \mathrm{~kg} / \mathrm{m}^{2}$ compared to $77 \%$ in those with BMI $<30$ $\mathrm{kg} / \mathrm{m}^{222}$
To address these problems, recently revised clinical HCC guidelines have allowed the use of alternative surveillance tools such as computed tomography (CT) and magnetic resonance imaging (MRI) in patients with inadequate surveillance on US. ${ }^{2-4}$ Recent studies have explored the potentials of $\mathrm{CT}$ and MRI with various techniques as alternative tools for HCC surveillance.

\section{IMAGING MODALITIES BEYOND US}

1. CT

CT for HCC surveillance is yet to be established due to the paucity of data. To our knowledge, only one prospective study ${ }^{23}$ has evaluated contrast-enhanced CT as an alternative surveillance tool by comparing its performance to that of US in 163 patients with 17 HCCs. The study concluded that biannual US was marginally more sensitive $(71.4 \%)$ and less costly compared to annual CT (66.7\%) for the detection of HCC. However, the limitations of that study included its unclear positivity criteria for each imaging modality, that only 10 of the 17 HCCs were early-stage, and the small number of patients and HCCs. An on-going prospective trial (A Prospective Cohort Study to Compare Contrast Enhanced Liver CT \& US for HCC Surveillance in High-risk Group of HCC. ClinicalTrials.gov NCT02675920) comparing contrast-enhanced CT to US for HCC surveillance in high-risk populations will hopefully provide valuable information regarding CT-based HCC surveillance. In a diagnostic setting, a recent meta-analysis ${ }^{24}$ reported an inferior sensitivity of CT to that of MRI ( $66 \%$ vs. $82 \%$ ), while the specificity of both modalities did not differ significantly (CT vs. MRI, $92 \%$ vs. $91 \%$ ). Although performances in a diagnostic setting cannot be directly applied in a surveillance setting, CT performance is expected to be lower than that of MRI for surveillance purpose. Regardless of the performance of CT for this purpose, due to the repetitive nature of surveillance examinations, the cumulated radiation hazard and potential risks related to the use of iodine contrast agents remain significant hurdles for the use of CT in a surveillance setting. 


\section{MRI}

\section{1) Full MRI with gadoxetic acid}

Gadoxetic acid-enhanced MRI showed superb diagnostic performance in diagnosing small HCCs $(2-3 \mathrm{~cm}){ }^{25-27}$ Gadoxetic acid-enhanced MRI with full sequences provides not only hemodynamic information as does extracellular contrast agents but also provides additional hepatobiliary phase (HBP) images which maximize focal lesion detection. According to a study comparing US and gadoxetic acid-enhanced MRI, the sensitivity of MRI (84.8\%) was significantly higher than that of US $(27.3 \%)(P<0.001)$ for detecting very early-stage HCC. ${ }^{18}$ Although gadoxetic acid-enhanced MRI with full sequences may be the best option from a performance point of view, the long acquisition time and high cost hamper its widespread use.

The diagnostic performance of gadoxetic acid-enhanced MRI may be affected by technical challenges and patient liver function. The optimal arterial phase images in liver MRI using gadoxetic acid are trickier to obtain compared to liver MRI using extracellular contrast agents because of the small administered volume and gadolinium content as well as the occurrence of transient severe motion. ${ }^{28}$ Hepatic parenchymal enhancement can become heterogeneous and diminished in patients with compromised liver function, which affects lesion conspicuity during the HBP. ${ }^{29}$ Thus, these could mitigate the sensitivity of MRI and also produce pseudolesions. To our knowledge, little has been reported about the effects of these factors on the performance of surveillance tests.

\section{2) Abbreviated MRI with gadoxetic acid or extracellu- lar contrast agent}

To reduce scan time and sequences, abbreviated MRI (AMRI) protocols comprising only selected sequences such as the HBP of gadoxetic acid-enhanced MRI $\mathrm{I}^{30-32}$ or dynamic contrast-enhanced sequences with extracellular contrast agents-enhanced $\mathrm{MRI}^{33}$ are currently areas of active research. AMRI with gadoxetic acid usually includes HBP, T1-weighted sequence, and diffusion-weighted imaging (DWI) for HCC detection, with T2-weighted sequences to improve specificity by excluding cysts or hemangiomas. AMRI with gadoxetic acid has a sensitivity of $80-90 \%$ and specificity of 91-98\% for detecting HCC. ${ }^{30-32}$ However, the results of these studies should be interpreted with caution. As the incidence of HCC in these studies was unrealistically high (up to $18.2 \%)$, there may have been selection bias in the study populations. An ongoing prospective trial in Korea aims to determine the clinical feasibility of AMRI with gadoxetic acid compared to US (Clinical Feasibility of Abbreviated MRI for HCC Surveillance in High-risk Group, ClinicalTrials.gov NCT03731923). However, if a lesion is detected on AMRI with gadoxetic acid, the lack of hemodynamic information necessities further examination with an additional contrastenhanced study with multiphase dynamic images to confirm whether the detected lesion is HCC. To avoid this additional imaging study, a retrospective study ${ }^{33}$ proposed a different approach using AMRI with extracellular agent containing only dynamic sequences, showing only $5 \%$ of changes in Liver Reporting \& Data System (LI-RADS) categorization based on interpretation only of AMRI with extracellular agent compared to that of full sequence MRI. AMRI with extracellular contrast agent required only 7-10 minutes of scan time, suggesting its potential usefulness. However, the study was limited as it did not report the diagnostic performances for very early-stage HCC and only reported changes in LI-RADS categorization. Similar to full MRI with gadoxetic acid, the image quality of AMRI using gadoxetic acid may be lower in patients with poor liver function, affecting the diagnostic performance of this modality; furthermore, little has been reported about the effects of these factors on surveillance tests. $^{29}$

\section{3) Increased awareness of the potential risks related to gadolinium-based MRI contrast agent}

Although gadolinium-based MRI contrast agents are safer than the iodinated contrast agents used in CT, recent studies have warned that, in addition to nephrogenic systemic fibrosis, gadolinium also accumulates in body tissue after repeated exposure. ${ }^{34,35}$ Thus, the use of a gadolinium contrast agent, whether in full MRI or AMRI, bears potential risks related to the retention of gadolinium in human tissue. 


\section{4) Non-contrast MRI}

Non-contrast MRI is free of gadolinium-related concerns. Non-contrast MRI composed of DWI and T2-weighted imaging may also be a promising option ${ }^{36,37}$ for HCC surveillance. DWI has shown excellent performance in detecting HCC, while T2-weighted imaging allows the exclusion of common benign lesions such as hemangiomas or cysts. The combination of DWI and T2-weighted imaging may be a practical surveillance strategy for HCC. A study comparing the performance of non-enhanced MRI and US for HCC surveillance in high-risk patients ${ }^{38}$ showed significantly higher per-lesion and per-exam sensitivities for non-enhanced MRI (77.1\% and 79.1\%, respectively) compared to those for US ( $25.0 \%$ and $27.9 \%$, respectively, $P<0.001)$. In this study, non-contrast MRI also showed significantly higher specificity than that of US ( $97.9 \%$ vs. $94.5 \%$, respectively, $P<0.001$ ). Two prospective studies in Korea are currently evaluating the usefulness of non-contrast MRI, namely, the Annual MRI Versus Biannual US for Surveillance of HCC in liver cirrhosis (MAGNUS-HCC, ClinicalTrials.gov NCT02551250) and Usefulness of Non-contrast MRI versus Non-Contrast US for Surveillance of HCC (MIRACLE-HCC, ClinicalTrials. gov NCT02514434).

\section{PERFLUOROBUTANE-ENHANCED US}

The introduction of a new US contrast agent, perfluorobutane gas-containing microbubbles (Sonazoid; GE Healthcare, Oslo, Norway), provides new opportunities for the use of contrast-enhanced US as a surveillance tool for HCC. ${ }^{39}$ It allows stable post-vascular or Kupffer phase imaging for more than 60 minutes as well as vascular phase imaging, which provides sufficient time to scan the whole liver. Two prospective studies tested the performance of perfluorobutaneenhanced $\mathrm{US}^{40,41}$ in a surveillance setting, with contradicting results. One study from Korea ${ }^{40}$ reported that the detection rate of early-stage HCC was not significantly improved by adding perfluorobutane-enhanced US to conventional Bmode US (difference, $0.4 \% ; P=0.16$ ), although the false referral rate was significantly reduced (difference, $-3.2 \%$; $P<0.001$ ). In contrast, a study from $\operatorname{Japan}^{41}$ suggested that perfluorobutane-enhanced US was useful for the early detection of HCC by showing significantly smaller HCC on contrast-enhanced US $(13.0 \mathrm{~mm})$ than that detected by conventional B-mode US $(16.7 \mathrm{~mm}, P=0.011)$. In addition, the inherent limitations of conventional B-mode US on poor sonic window for advanced cirrhotic liver cannot be com-

Table 1. Characteristics of studies assessing the performance of advanced imaging modalities for HCC surveillance

\begin{tabular}{|c|c|c|c|c|c|c|c|c|}
\hline Image modality & Study & Country & $\begin{array}{l}\text { Study } \\
\text { period }\end{array}$ & Study design & $\begin{array}{c}\text { Number } \\
\text { of } \\
\text { patients }\end{array}$ & $\begin{array}{l}\text { Number of } \\
\text { patients } \\
\text { with HCC }\end{array}$ & $\begin{array}{c}\text { Sensitivity } \\
(\%)\end{array}$ & $\begin{array}{c}\text { Specificity } \\
(\%)\end{array}$ \\
\hline Dynamic CT & Pocha et al. ${ }^{23}$ & USA & $2002-2011$ & Prospective & 163 & $17(10.4)$ & 66.7 & 94.4 \\
\hline $\begin{array}{l}\text { Full MRI with gadoxetic } \\
\text { acid }\end{array}$ & Kim et al. ${ }^{18}$ & Korea & 2011-2014 & Prospective & 407 & $37(9.1)^{*}$ & $84.8^{*}$ & $\mathrm{~N} / \mathrm{A}$ \\
\hline \multirow{2}{*}{$\begin{array}{l}\text { Abbreviated MRI with } \\
\text { gadoxetic acid }\end{array}$} & Besa et al. ${ }^{31}$ & USA & 2011 & Retrospective & 340 & $62(18.2)$ & $85.5-90.3$ & 84.8-100 \\
\hline & Tillman et al. ${ }^{32}$ & USA & 2008-2014 & Retrospective & 79 & $13(16.5)$ & $85.2^{\dagger}$ & $\mathrm{N} / \mathrm{A}$ \\
\hline \multirow[t]{2}{*}{ Non-contrast MRI } & Kim et al. ${ }^{36}$ & Korea & 2010-2012 & Retrospective & 135 & $128^{\ddagger}$ & $91.7^{\dagger \neq}$ & $77.5^{\dagger \neq}$ \\
\hline & Park et al. ${ }^{38}$ & Korea & 2011-2014 & Retrospective & 382 & $43(11.3)$ & 79.1 & 97.9 \\
\hline \multirow{2}{*}{$\begin{array}{l}\text { Perfluorobutane- } \\
\text { enhanced US }\end{array}$} & Park et al. ${ }^{40}$ & Korea & 2014-2016 & Prospective & 524 & $6(1.1)$ & $100.0^{\S}$ & N/A \\
\hline & Kudo et al. ${ }^{41}$ & Japan & $\mathrm{N} / \mathrm{A}$ & Prospective & 309 & $52(16.8)$ & 100.0 & 96.1 \\
\hline
\end{tabular}

Unless otherwise indicated, sensitivity and specificity are per-patient data. Values are presented as number (\%) unless otherwise indicated. HCC, hepatocellular carcinoma; CT, computed tomography; MRI, magnetic resonance imaging; N/A, not available; US, ultrasound.

*For very early-stage HCC; ${ }^{\dagger}$ Per-lesion data; ${ }^{\ddagger}$ Including HCC and intrahepatic cholangiocarcinoma; ${ }^{5}$ For early-stage HCC. 
pletely mitigated on contrast-enhanced US. Perfluorobutane-enhanced US may be an option for HCC surveillance among advanced imaging modalities, although additional studies are warranted to support its usefulness.

\section{COST-EFFECTIVENESS}

In a surveillance setting, the availability and cost-effectiveness of these advanced imaging modalities also require consideration. ${ }^{42}$ The key factors determining their cost-effectiveness for cancer screening include not only the cost of diagnostic tests but also the diagnostic performances of imaging studies, the incidence of HCC, and the amount of survival gained by treatment. ${ }^{43-45}$ Two studies demonstrated that risk-stratified HCC surveillance strategies with MRI targeting high- and intermediate-risk patients with cirrhosis were costeffective and outperformed the currently recommended nonstratified biannual US in all patients with cirrhosis. ${ }^{46,47}$ Goossens et al. ${ }^{46}$ reported the lowest incremental cost-effective ratio of $\$ 2,100$ per quality-adjusted life-year for the use of
AMRI for high- and intermediate-risk patients without screening of low-risk patients. Another study using Markov modeling suggested that full gadoxetic acid-enhanced MRI might be a cost-effective surveillance option in high-risk patients with an annual HCC incidence rate of $1.81 \%$ or higher, with an incremental cost-effective ratio of less than $\$ 50,000$ per quality-adjusted life-year. ${ }^{47}$

\section{CONCLUSIONS}

Evidence of the clinical feasibility of advanced imaging modalities other than US is rapidly accumulating owing to efforts to overcome the limitations of US for HCC surveillance, especially for very early-stage HCC (Table 1). The various options in advanced imaging modalities for HCC surveillance have their strengths and weaknesses (Table 2) which should be considered when determining the most appropriate niche for these new approaches. Instead of a one-size-fitsall approach with biannual US regardless of the HCC risks of individual patients, the results of recent studies support a

Table 2. Summary of the strengths and weaknesses of each imaging modality for HCC surveillance

\begin{tabular}{|c|c|c|}
\hline Imaging modality & Strength & Weakness \\
\hline Dynamic CT & $\begin{array}{l}\text { Short scan time } \\
\text { Assessment of HCC hallmark }\end{array}$ & $\begin{array}{l}\text { Accumulative radiation hazard } \\
\text { Potential harms related to the contrast agent }\end{array}$ \\
\hline Full MRI with gadoxetic acid & $\begin{array}{l}\text { The best diagnostic performance } \\
\text { Assessment of HCC hallmark } \\
\text { No radiation hazard }\end{array}$ & $\begin{array}{l}\text { Long scan and interpretation times } \\
\text { High cost } \\
\text { Potential harms related to the contrast agent }\end{array}$ \\
\hline $\begin{array}{l}\text { Abbreviated MRI with gadoxetic } \\
\text { acid }\end{array}$ & $\begin{array}{l}\text { Shorter scan time than that of full MRI } \\
\text { Equivalent sensitivity to that of full MRI } \\
\text { No radiation hazard }\end{array}$ & $\begin{array}{l}\text { No assessment of HCC hallmark } \\
\text { Potential harms related to the contrast agent }\end{array}$ \\
\hline $\begin{array}{l}\text { Abbreviated MRI with extracellular } \\
\text { contrast agent }\end{array}$ & $\begin{array}{l}\text { Shorter scan time than that of full MRI } \\
\text { Minimal changes in LI-RADS categorization } \\
\text { Assessment of HCC hallmark } \\
\text { No radiation hazard }\end{array}$ & Potential harms related to the contrast agent \\
\hline Non-contrast MRI & $\begin{array}{l}\text { Shorter scan time than those of full or abbreviated } \\
\text { MRI } \\
\text { No harm related to the contrast agent } \\
\text { No radiation hazard }\end{array}$ & No assessment of HCC hallmark \\
\hline Perfluorobutane-enhanced US & $\begin{array}{l}\text { Real-time vascular imaging and long-lasting Kupffer } \\
\text { phase imaging } \\
\text { No radiation hazard } \\
\text { Assessment of HCC hallmark }\end{array}$ & $\begin{array}{l}\text { Inherent limitation of conventional B-mode US on } \\
\text { poor sonic window for advanced cirrhotic liver }\end{array}$ \\
\hline
\end{tabular}

HCC, hepatocellular carcinoma; CT, computed tomography; MRI, magnetic resonance imaging; LI-RADS, Liver Imaging Reporting And Data System; US, ultrasound. 
risk-stratified approach with advanced imaging modalities. The potential harms related to these alternative approaches and the availability of medical resources should also be considered. ${ }^{48}$

\section{Conflicts of Interest}

The authors declare no conflicts of interest pertaining to this work.

\section{REFERENCES}

1. Kim NM, Doh YS, Jang JW, Kim SH, Eun HS, Jun JH, et al. Discrepancy between the actual clinical status of patients with hepatocellular carcinoma and expectations from hepatocellular carcinoma surveillance: a single-center study. J Liver Cancer 2019;19:30-37.

2. European Association for the Study of the Liver. EASL Clinical Practice Guidelines: management of hepatocellular carcinoma. J Hepatol 2018;69:182-236.

3. Marrero JA, Kulik LM, Sirlin CB, Zhu AX, Finn RS, Abecassis MM, et al. Diagnosis, staging, and management of hepatocellular carcinoma: 2018 Practice Guidance by the American Association for the Study of Liver Diseases. Hepatology 2018;68:723-750.

4. Korean Liver Cancer Association, National Cancer Center. 2018 Korean Liver Cancer Association-National Cancer Center Korea practice guidelines for the management of hepatocellular carcinoma. Gut Liver 2019;13:227-299.

5. Park JW, Chen M, Colombo M, Roberts LR, Schwartz M, Chen PJ, et al. Global patterns of hepatocellular carcinoma management from diagnosis to death: the BRIDGE Study. Liver Int 2015;35:21552166.

6. Yang JD. Detect or not to detect very early stage hepatocelIular carcinoma? The western perspective. Clin Mol Hepatol 2019;25:335-343.

7. Hwang S, Lee YJ, Kim KH, Ahn CS, Moon DB, Ha TY, et al. The impact of tumor size on long-term survival outcomes after resection of solitary hepatocellular carcinoma: single-institution experience with 2558 patients. J Gastrointest Surg 2015;19:1281-1290.

8. Tabrizian P, Jibara G, Shrager B, Schwartz M, Roayaie S. Recurrence of hepatocellular cancer after resection: patterns, treatments, and prognosis. Ann Surg 2015;261:947-955.

9. Groeschl RT, Gamblin TC, Turaga KK. Ablation for hepatocelIular carcinoma: validating the $3-\mathrm{cm}$ breakpoint. Ann Surg Oncol 2013;20:3591-3595.

10. Brunt E, Aishima S, Clavien PA, Fowler K, Goodman Z, Gores G, et al. CHCC-CCA: Consensus terminology for primary liver carcinomas with both hepatocytic and cholangiocytic differentation. Hepatology 2018;68:113-126.

11. Livraghi T, Meloni F, Di Stasi M, Rolle E, Solbiati L, Tinelli C, et al. Sustained complete response and complications rates after radiofrequency ablation of very early hepatocellular carcinoma in cirrhosis: is resection still the treatment of choice? Hepatology 2008;47:82-89.

12. Takayama T, Makuuchi M, Hirohashi S, Sakamoto M, Yamamoto J, Shimada K, et al. Early hepatocellular carcinoma as an entity with a high rate of surgical cure. Hepatology 1998;28:1241-1246.

13. Shindoh J, Andreou A, Aloia TA, Zimmitti G, Lauwers GY, Laurent $A$, et al. Microvascular invasion does not predict long-term survival in hepatocellular carcinoma up to $2 \mathrm{~cm}$ : reappraisal of the staging system for solitary tumors. Ann Surg Oncol 2013;20:1223-1229.

14. Goldberg SN, Grassi CJ, Cardella JF, Charboneau JW, Dodd GD 3rd, Dupuy DE, et al. Image-guided tumor ablation: standardization of terminology and reporting criteria. J Vasc Interv Radiol 2009;20(7 Suppl):S377-S390.

15. Wong LL, Reyes RJ, Kwee SA, Hernandez BY, Kalathil SC, Tsai NC. Pitfalls in surveillance for hepatocellular carcinoma: how successful is it in the real world? Clin Mol Hepatol 2017;23:239-248.

16. Singal A, Volk ML, Waljee A, Salgia R, Higgins P, Rogers MA, et al. Meta-analysis: surveillance with ultrasound for early-stage hepatocellular carcinoma in patients with cirrhosis. Aliment Pharmacol Ther 2009;30:37-47.

17. Tzartzeva K, Obi J, Rich NE, Parikh ND, Marrero JA, Yopp A, et al. Surveillance imaging and alpha fetoprotein for early detection of hepatocellular carcinoma in patients with cirrhosis: a metaanalysis. Gastroenterology 2018;154:1706-1718.e1701.

18. Kim SY, An J, Lim YS, Han S, Lee JY, Byun JH, et al. MRI with liverspecific contrast for surveillance of patients with cirrhosis at high risk of hepatocellular carcinoma. JAMA Oncol 2017;3:456-463.

19. Simmons O, Fetzer DT, Yokoo T, Marrero JA, Yopp A, Kono Y, et al. Predictors of adequate ultrasound quality for hepatocellular carcinoma surveillance in patients with cirrhosis. Aliment Pharmacol Ther 2017;45:169-177.

20. Son JH, Choi SH, Kim SY, Jang HY, Byun JH, Won HJ, et al. Validation of US Liver Imaging Reporting and Data System Version 2017 in patients at high risk for hepatocellular carcinoma. Radiology 2019;292:390-397.

21. Yu NC, Chaudhari V, Raman SS, Lassman C, Tong MJ, Busuttil RW, et al. CT and MRI improve detection of hepatocellular carcinoma, compared with ultrasound alone, in patients with cirrhosis. Clin Gastroenterol Hepatol 2011;9:161-167.

22. Esfeh JM, Hajifathalian K, Ansari-Gilani K. Sensitivity of ultrasound in detecting hepatocellular carcinoma in obese patients compared to explant pathology as the gold standard. Clin Mol Hepatol 2020;26:54-59.

23. Pocha C, Dieperink E, McMaken KA, Knott A, Thuras P, Ho SB. Surveillance for hepatocellular cancer with ultrasonography vs. computed tomography -- a randomised study. Aliment Pharmacol Ther 2013;38:303-312.

24. Roberts LR, Sirlin CB, Zaiem F, Almasri J, Prokop LJ, Heimbach JK, 
et al. Imaging for the diagnosis of hepatocellular carcinoma: a systematic review and meta-analysis. Hepatology 2018;67:401-421.

25. Kierans AS, Kang SK, Rosenkrantz AB. The diagnostic performance of dynamic contrast-enhanced MR imaging for detection of small hepatocellular carcinoma measuring up to $2 \mathrm{~cm}$ : a meta-analysis. Radiology 2016;278:82-94.

26. Chou R, Cuevas C, Fu R, Devine B, Wasson N, Ginsburg A, et al. Imaging techniques for the diagnosis of hepatocellular carcinoma: a systematic review and meta-analysis. Ann Intern Med 2015;162:697-711.

27. Choi SH, Byun JH, Lim YS, Yu E, Lee SJ, Kim SY, et al. Diagnostic criteria for hepatocellular carcinoma $\leq 3 \mathrm{~cm}$ with hepatocytespecific contrast-enhanced magnetic resonance imaging. J Hepatol 2016;64:1099-1107.

28. Huh J, Kim SY, Yeh BM, Lee SS, Kim KW, Wu EH, et al. Troubleshooting arterial-phase MR images of gadoxetate disodiumenhanced liver. Korean J Radiol 2015;16:1207-1215.

29. Kim SY, Wu EH, Park SH, Wang ZJ, Hope TA, Yee J, et al. Comparison of hepatocellular carcinoma conspicuity on hepatobiliary phase images with gadoxetate disodium vs. delayed phase images with extracellular cellular contrast agent. Abdom Radiol (NY) 2016;41:1522-1531.

30. Marks RM, Ryan A, Heba ER, Tang A, Wolfson TJ, Gamst AC, et al. Diagnostic per-patient accuracy of an abbreviated hepatobiliary phase gadoxetic acid-enhanced MRI for hepatocellular carcinoma surveillance. AJR Am J Roentgenol 2015;204:527-535.

31. Besa C, Lewis S, Pandharipande PV, Chhatwal J, Kamath A, Cooper $\mathrm{N}$, et al. Hepatocellular carcinoma detection: diagnostic performance of a simulated abbreviated MRI protocol combining diffusion-weighted and T1-weighted imaging at the delayed phase post gadoxetic acid. Abdom Radiol (NY) 2017;42:179-190.

32. Tillman BG, Gorman JD, Hru JM, Lee $M H$, King MC, Sirlin CB, et al. Diagnostic per-lesion performance of a simulated gadoxetate disodium-enhanced abbreviated MRI protocol for hepatocellular carcinoma screening. Clin Radiol 2018;73:485-493.

33. Lee JY, Huo EJ, Weinstein S, Santos C, Monto A, Corvera CU, et al. Evaluation of an abbreviated screening MRI protocol for patients at risk for hepatocellular carcinoma. Abdom Radiol (NY) 2018;43:1627-1633.

34. Rogosnitzky M, Branch S. Gadolinium-based contrast agent toxicity: a review of known and proposed mechanisms. Biometals 2016;29:365-376.

35. Levine D, McDonald RJ, Kressel HY. Gadolinium retention after contrast-enhanced MRI. JAMA 2018;320:1853-1854.

36. Kim YK, Kim YK, Park HJ, Park MJ, Lee WJ, Choi D. Noncontrast MRI with diffusion-weighted imaging as the sole imaging modality for detecting liver malignancy in patients with high risk for hepatocellular carcinoma. Magn Reson Imaging 2014;32:610-618.

37. Han S, Choi JI, Park MY, Choi MH, Rha SE, Lee YJ. the diagnostic performance of liver MRI without intravenous contrast for detecting hepatocellular carcinoma: a case-controlled feasibility study. Korean J Radiol 2018;19:568-577.

38. Park HJ, Jang HY, Kim SY, Lee SJ, Won HJ, Byun JH, et al. Nonenhanced magnetic resonance imaging as a surveillance tool for hepatocellular carcinoma: comparison with ultrasound. J Hepatol 2020;72:718-724.

39. Maruyama H, Shiina S. Contrast-enhanced ultrasonography: is it an ideal tool for hepatocellular carcinoma surveillance? Quant Imaging Med Surg 2019;9:1611-1614.

40. Park JH, Park MS, Lee SJ, Jeong WK, Lee JY, Park MJ, et al. Contrast-enhanced US with perfluorobutane for hepatocellular carcinoma surveillance: a multicenter diagnostic trial (SCAN). Radiology 2019;292:638-646.

41. Kudo M, Ueshima K, Osaki Y, Hirooka M, Imai Y, Aso K, et al. BMode ultrasonography versus contrast-enhanced ultrasonography for surveillance of hepatocellular carcinoma: a prospective multicenter randomized controlled trial. Liver Cancer 2019;8:271-280.

42. Su TH, Kao JH. Surveillance of hepatocellular carcinoma by magnetic resonance imaging with liver-specific contrast. JAMA Oncol 2017;3:446-447.

43. Cucchetti A, Trevisani F, Cescon M, Ercolani G, Farinati F, Poggio $P D$, et al. Cost-effectiveness of semi-annual surveillance for hepatocellular carcinoma in cirrhotic patients of the Italian Liver Cancer population. J Hepatol 2012;56:1089-1096.

44. Arguedas MR, Chen VK, Eloubeidi MA, Fallon MB. Screening for hepatocellular carcinoma in patients with hepatitis $C$ cirrhosis: a cost-utility analysis. Am J Gastroenterol 2003;98:679-690.

45. Ruggeri M. Hepatocellular carcinoma: cost-effectiveness of screening. A systematic review. Risk Manag Healthc Policy 2012;5:49-54.

46. Goossens N, Singal AG, King LY, Andersson KL, Fuchs BC, Besa $C$, et al. Cost-effectiveness of risk score-stratified hepatocellular carcinoma screening in patients with cirrhosis. Clin Transl Gastroenterol 2017;8:e101.

47. Kim HL, An J, Park JA, Park SH, Lim YS, Lee EK. Magnetic resonance imaging is cost-effective for hepatocellular carcinoma surveillance in high risk patients with cirrhosis. Hepatology 2019;69:1599-1613.

48. Atiq 0, Tiro J, Yopp AC, Muffler A, Marrero JA, Parikh ND, et al. An assessment of benefits and harms of hepatocellular carcinoma surveillance in patients with cirrhosis. Hepatology 2017;65:11961205. 